\title{
Diagnosis and Management of Post Transfusion Purpura: A Case Report
}

Nosha Farhadfar*, Amishi Desai, Abirami Sivapiragasam, Joshua Harris, Anish Desai and Alexander Hindenburg

Medical Oncology and Hematology, Winthrop University Hospital, New York, USA

\begin{abstract}
Post-Transfusion Purpura (PTP) is a rare, yet potentially fatal bleeding disorder characterized by severe thrombocytopenia, purpura and hemorrhage that occurs after transfusion of blood product. It is caused by alloimmunization against platelet antigens, anti-HPA-1a being the most frequent antibody. We report a rare case of PTP due to an even more unusual anti-HPA-5a alloantibody after platelet transfusion and discuss the clinical presentation, diagnosis and management of this life threatening condition.
\end{abstract}

Keywords: Thrombocytopenia; Transfusion; Purpura

\section{Introduction}

PTP is a rare syndrome which was first described by Shulman et al in 1961. This immuno-hematologic disorder is characterized by lifethreatening thrombocytopenia occurring 7 to 14 days after transfusion of blood products. Severe thrombocytopenia in PTP is due to an alloantibody against donor platelet antigen, most commonly human platelet antigen-1a (HPA-1a), an epitope on glycoprotein IIIa of GP IIb/ IIIa complex [1]. The recipients always have a history of sensitization, mostly by pregnancy, and occasionally by blood transfusion. The femaleto-male ratio is 5:1. The incidence of PTP is approximately 1 in 50000100000 transfusions [1,2]. In untreated patient, thrombocytopenia resolves in approximately 20 days [3]. However, bleeding from mucous membranes, gastrointestinal and urinary tract is common and mortality rate has been estimated to be approximately $10 \%$, which is mostly secondary to intracranial hemorrhage [2]. Therefore, prompt diagnosis and initiation of appropriate therapy in essential to decrease the mortality associated with this disorder.

This case represents one of the very few PTP reported in literature with alloantibody against human platelet antigen-5a (HPA-5a) managed in our facility. Only $1.1 \%$ of the population is homozygous for HPA-5b, hence the low incident of PTP related to anti HPA-5a [2,4].

\section{Case Summary}

80 -year-old woman with severe aortic stenosis and coronary artery disease was admitted for valve replacement and coronary artery bypass graft (CABG) surgery. Her surgery was uncomplicated, but multiple blood products were given perioperatively. Platelet count declined from $140 \times 10^{9} / \mathrm{L}$ to $80 \times 10^{9} / \mathrm{L}$ one-day post surgery. This transient decline in platelet count is commonly seen post-cardiac surgery and has been attributed to mechanical consumption of platelets by the bypass machine and takes up to 10 days for recovery. Patient also developed a mediastinal hematoma requiring evacuation and multiple units of platelet transfusions two days after surgery. She was started on intravenous antibiotics (vancomycin and cefepime) for fever of unclear etiology. Given, further decline in platelet count despite platelet transfusion other aetiologies for thrombocytopenia was explored.

Review of peripheral blood film confirmed marked reduction in platelets with no evidence of microangiopathy. Due to recent exposure to heparin and a positive heparin antibody test, argatroban was started empirically for possible heparin induced thrombocytopenia (HIT). However, there was no resultant improvement in platelet count and the level acutely dropped reaching a nadir of $6 \times 10^{9} / \mathrm{L}$ after subsequent platelet transfusion. Patient developed petechial rash on trunk on postoperative day 14 (Figure 1).

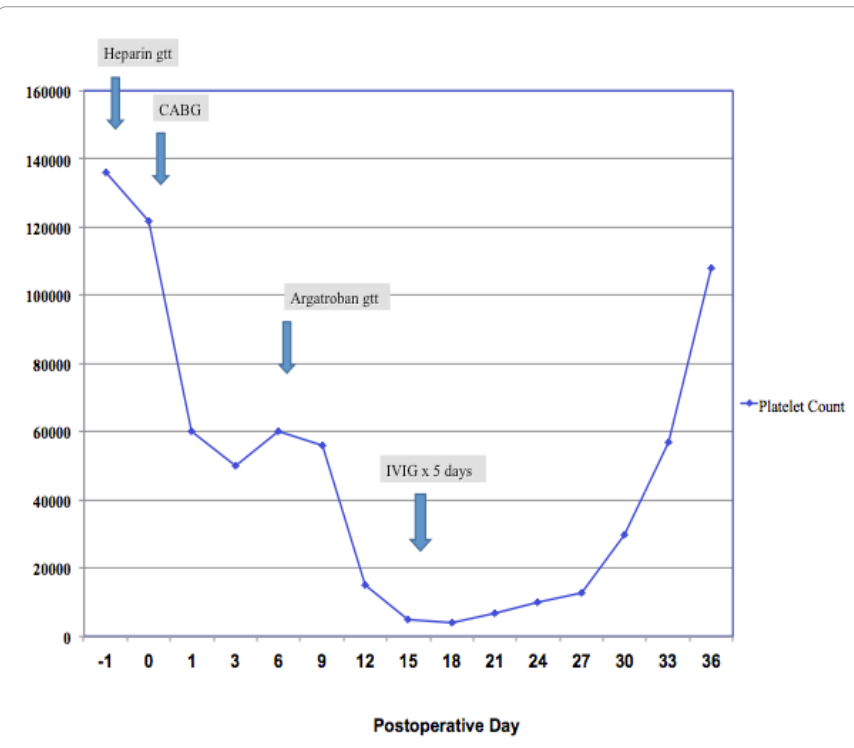

Figure 1: Graph showing platelet count trend and interventions.

PTP was suspected due to presence of profound thrombocytopenia with petechial rash, therefore platelet antibody assay was sent. Argatroban was stopped after development of inguinal hematoma; an unusual occurrence in HIT and empiric therapy for PTP was initiated with $0.5 \mathrm{mg} / \mathrm{kg}$ of Intravenous Immunoglobulin (IVIG). Platelet count started to rise after 5 days of IVIG. An antibody with reactivity to HPA$5 \mathrm{a}$ was detected by enzyme-linked immunoassay and the patient was found to be homozygous for HPA-5b by genotyping. One month later, the patient's platelet count normalized to $193 \times 10^{9} / \mathrm{L}$.

\section{Discussion}

We have described the clinical course of a patient with a challenging diagnosis of PTP. This was supported by serologic studies demonstrating

*Corresponding author: Nosha Farhadfar, Medical Oncology and Hematology, Winthrop University Hospital, New York, USA, Tel: +1 516-663-0333; E-mail: amishi198@gmail.com

Received April 25, 2015; Accepted June 07, 2015; Published June 09, 2015

Citation: Farhadfar N, Desai A, Sivapiragasam A, Harris J, Desai A, et al. (2015) Diagnosis and Management of Post Transfusion Purpura: A Case Report. J Clin Case Rep 5: 550. doi:10.4172/2165-7920.1000550

Copyright: (c) 2015 Farhadfar N, et al. This is an open-access article distributed under the terms of the Creative Commons Attribution License, which permits unrestricted use, distribution, and reproduction in any medium, provided the original author and source are credited. 
an alloantibody directed against HPA-5a and genotyping indicating that the patient was homozygous for HPA-5b.

PTP appears in patients pre-exposed to foreign platelet-specific antigens, who then develop high titers of anti-HPA antibodies following transfusion of blood products. These antibodies paradoxically destroy recipient platelets and the proposed mechanisms of this destruction are: adsorption of antigen-antibody complexes, cross-reactive antibodies or autoantibody production [1-5].

The majority of PTP cases occur in patients with HPA-1b/b genotype producing anti-HPA-1a, however the offending antibodies can be directed against HPA-3a, 3b, 4a, 5a and 5b. The allelic frequency of HPA-5b is only $0.11 \%$, and only $1.11 \%$ of population is homozygous for HPA-5b. The HPA-5a antigens are expressed at a much lower density and are located on glycoprotein Ia of the GPIa/IIa complex, which can mediate adhesion to collagen. HPA- 5 system has been recognized as the second most frequent alloantibody involved in neonatal alloimmnune thrombocytopenia [4].

Despite the dramatic clinical presentation of extensive bleeding due to a low number of platelets, PTP is a self-limited disorder. However, given the high fatality rate of about $10-20 \%$ early in the course of the disease, correct diagnosis and immediate treatment is essential.

Due to similar clinical presentation of Heparin induced thrombocytopenia and PTP, differentiating between these two entities can be challenging. Management of the patient can also be misguided by a false positive HIT-antibody screening assay as shown in this case. Although thrombocytopenia is a common feature of HIT, platelet count value of less than $20 \times 10^{9} / \mathrm{L}$ is rarely seen in HIT, but is common in PTP [4-7]. In addition, HIT has been associated with thrombosis rather than bleeding. Therefore, presence of severe thrombocytopenia (platelet count less than $20 \times 10^{9} / \mathrm{L}$ ), petechia, and recent blood product transfusion should favours diagnosis of PTP $[6,7]$.

Use of plasma exchange in treatment of PTP is controversial. The treatment of choice is immunomodulation by administration of 0.5 $\mathrm{mg} / \mathrm{kg}$ IVIG for 5 days with or without corticosteroids [1,8]. Although some published reports suggest that patients do not respond to platelet transfusions, in rare cases of life-threatening hemorrhage, transfusion of platelets lacking the "guilty antigen" may temporarily increase the platelet number, stop the bleeding and save lives.

\section{Conclusion}

This case highlights the importance of maintaining a high level of suspicion for PTP in the differential while evaluating life-threatening thrombocytopenia in both men and women with a recent history of blood transfusion. Prompt diagnosis and initiation of appropriate therapy can shorten the duration of thrombocytopenia and reduce both morbidity and mortality.

\section{References}

1. Rozman $P$ (2002) Platelet antigens. The role of human platelet alloantigens (HPA) in blood transfusion and transplantation. Transpl Immunol 10: 165-181.

2. Anolik JH, Blumberg N, Snider J, Francis CW (2001) Posttransfusion purpura secondary to an alloantibody reactive with HPA-5a $(\operatorname{Br}(\mathrm{b}))$. Transfusion 41: 633636.

3. Vogelsang G, Kickler TS, Bell WR (1986) Post-transfusion purpura: a report of five patients and a review of the pathogenesis and management. Am J Hematol 21: 259-267.

4. Christie DJ, Pulkrabek S, Putnam JL, Slatkoff ML, Pischel KD (1991) Posttransfusion purpura due to an alloantibody reactive with glycoprotein la/lla (anti-HPA-5b). Blood 77: 2785-2789.

5. Araujo F, Sa JJ, Araujo V, Lopes M, Cunha-Ribeiro LM (2000) Post-transfusion purpura vs. heparin-induced thrombocytopenia: differential diagnosis in clinical practice. Transfusion Medicine 10: 323-324.

6. Lube now N, Eichler P, Albrecht D (2000) Very low platelet counts in posttransfusion purpura falsely diagnosed as heparin-induced thrombocytopenia. Report of four cases and review of literature. Thrombus Res 100: 115-125.

7. Hron G, Knutson F, Thiele T, Althaus K, Busemann C, et al. (2013) Alternative diagnosis to heparin-induced thrombocytopenia in two critically ill patients despite a positive PF4/heparin-antibody test. Ups J Med Sci 118: 279-284.

8. Ziman A, Klapper E, Pepkowitz S, Smith R, Garratty G, et al. (2002) A second case of post-transfusion purpura caused by HPA-5a antibodies: successful treatment with intravenous immunoglobulin. Vox Sang 83: 165-166. 